ACTUALIDAD

CLINICAL OVERVIEW

\title{
Estrategias de Prevención y Tratamiento en Displasia Broncopulmonar
}

\author{
ALDO BANCALARI M. ${ }^{1}$ \\ 1. Profesor Asociado de Pediatría. Director Programa de Neonatología, Departamento de Pediatría, Facultad de Medicina, \\ Universidad de Concepción.
}

\begin{abstract}
Strategies for Prevention and Treatment of Broncopulmonary Dysplasia
\end{abstract}

Bronchopulmonary Dysplasia (BPD) continues to be a highly frequent sequela of low birth weight infants. This, despite the many recent advances in perinatal medicine that include antenatal steroids, exogenous surfactant, new strategies for mechanical respiratory support, parenteral nutrition improvements and a more judicious use of supplemental oxygen. The purpose of this review is to analyze and describe the recent advances in the prevention and management of BPD. New preventive therapies have been developed, and vitamin A and caffeine administration have been shown to diminish the incidence of this disorder. Postnatal corticosteroids can also be useful, but due to their negative long-term neurological effects, these medications are not currently recommended. Corticosteroid administration in established BPD can decrease the need for mechanical ventilation and improve lung function. Other preventive interventions such as antioxidant administration and nitric oxide inhalation are currently being investigated. The persistence of the ductus arteriosus is associated with increased risk of BPD, therefore early pharmacologic closure can play a role in preventing this disorder. New modalities of mechanical ventilation, such as synchronized and high frequency ventilation, have not been shown to decrease the incidence of this disease. While a discussion regarding the best type of ventilation to be used still remains, the consensus is that that the lowest inspiratory pressure and lowest oxygen concentration necessary should be utilized to maintain adequate gas exchange. Optimal level of arterial oxygen saturation in premature infants is still controversial, but the current recommended range is between $88 \%$ and $95 \%$. Summary: Vitamin A and caffeine are both effective drugs in the prevention of BPD. The role of early closure of the ductus arteriosus and the use of postnatal administration of corticosteroids in the prevention of BPD are still not clearly defined.

(Key words: Bronchopulmonary dysplasia, prematurity, oxygen therapy, surfactant, mechanical ventilation, caffeine, corticosteroids).

Rev Chil Pediatr 2009; 80 (4): 309-322

\section{RESUMEN}

La Displasia Broncopulmonar (DBP) es una de las secuelas más frecuentes que afecta al recién nacido de muy bajo peso. Esto es, a pesar de los avances de la medicina perinatal de los últimos años, como la

Trabajo recibido el 05 de marzo de 2009, aceptado para publicación el 21 de mayo de 2009.

Correspondencia a:

Dr. Aldo Bancalari M.

E-mail: abancalari@entelchile.net 
administración de corticoides antenatales, surfactante exógeno, nuevos modos y estrategias de ventilación mecánica, mejoría de la nutrición parenteral y el uso más cuidadoso del oxígeno. El objetivo de la presente revisión es analizar y describir los recientes avances en la prevención y tratamiento de la DBP. Nuevas terapias preventivas han emergido, habiéndose demostrado que la administración de vitamina A y cafeína disminuyen la incidencia de esta afección. Los corticoides postnatales también disminuyen la incidencia de esta enfermedad, pero por sus efectos neurológicos a largo plazo, no se recomiendan actualmente. La administración de corticoides en la DBP establecida reduce el uso de ventilación mecánica y mejora la función pulmonar del recién nacido. Otras intervenciones preventivas como la administración de antioxidantes y óxido nítrico inhalado están siendo estudiadas. La persistencia del ductus arterioso se ha asociado a displasia, por lo cual, el cierre farmacológico precoz podría tener relevancia en la prevención de esta. Los nuevos modos de ventilación mecánica, como la ventilación sincronizada y la ventilación de alta frecuencia, no han disminuido la incidencia de esta enfermedad. Cualquiera sea el tipo de ventilación utilizada, debe aplicarse con la menor presión inspiratoria y concentración de oxígeno requeridas, para mantener un adecuado intercambio gaseoso. El rango de saturación arterial de oxígeno recomendado para el niño prematuro, aún es motivo de estudio, pero la recomendación actual más ampliamente aceptada es aquella que oscila entre $88 \%$ y 95\%. Conclusión: La vitamina A y la cafeína son drogas efectivas en la prevención de la DBP. Faltan estudios para determinar con mayor exactitud el posible rol del cierre temprano del ductus arterioso persistente y de la administración de esteroides post natales en la prevención de esta enfermedad.

(Palabras clave: Displasia broncopulmonar, prematuridad, oxígenoterapia, surfactante, ventilación mecánica, cafeína, corticoides).

Rev Chil Pediatr 2009; 80 (4): 309-322

\section{Introducción}

La displasia broncopulmonar (DBP) es una de las secuelas más frecuentes que afecta al recién nacido de muy bajo peso ${ }^{1,2}$; aproximadamente dos tercios de los prematuros, que desarrollan esta enfermedad son de extremo bajo peso (menor $1000 \mathrm{~g})^{1,2}$. A pesar de los avances de la medicina perinatal y neonatal que incluyen principalmente la administración de corticoides antenatales en partos prematuros y el tratamiento precoz con surfactante del recién nacido $(\mathrm{RN})$ con distrés respiratorio, la incidencia de DBP se ha mantenido sin variaciones $^{3,4}$, debido entre otros factores al aumento en la sobrevida de los RN muy inmaduros ${ }^{3-5}$.

En las últimas dos décadas han habido importantes avances en la comprensión de la patogénesis de esta enfermedad ${ }^{5}$, no ocurriendo lo mismo con respecto de las intervenciones destinadas a la prevención o tratamiento de la DBP, ya que muchas de ellas no están basadas en la evidencia ${ }^{6,7}$.

Las estrategias de prevención pueden ser prenatales $\mathrm{y} / \mathrm{o}$ postnatales. Las prenatales están bastante establecidas en el manejo médico habitual, las cuales son efectivas al realizarlas de manera adecuada ${ }^{8-10}$. No obstante las estrategias de prevención postnatales están en pleno desarrollo e investigación, algunas de las cuales se han confirmado científicamente y otras aún están en etapa experimental. Por tanto, de acuerdo a la evidencia científica estas se pueden dividir en estrategias probadas y no probadas (tabla 1).

El propósito de la presente revisión es actualizar los recientes avances en la prevención y tratamiento de la DBP.

\section{Estrategias de Prevención Antenatales}

\section{Prevención del Parto Prematuro}

Al evitar el parto prematuro, se previene la prematuridad y el distrés respiratorio, y por tanto la presencia de un niño a riesgo de desarrollar DBP. Este enfoque es sin duda la forma más eficaz de prevenir la DBP. Sin embargo, la disminución del parto prematuro ha sido un tema complejo y difícil de resolver ${ }^{8}$. La mayoría de las intervenciones orientadas para prevenir el parto prematuro no han funcionado ${ }^{8}$. No 
Tabla 1. Estrategias postnatales de prevención en displasia broncopulmonar

Probadas
- Soporte nutricional adecuado desde el nacimiento (vita-
mina A)
- Administración de cafeína
- Administración de corticoides
No probadas
- Manejo conservador y cuidadoso de las maniobras de
reanimación
- Administración precoz de surfactante exógeno
- Uso racional del oxígeno
- Uso precoz de CPAP nasal
- Ventilación mecánica mínima o conservadora
- Administración de antioxidantes exógenos
- Cierre precoz del ductus arterioso y restricción de líqui-
dos
- Óxido nítrico inhalado
- Administración de eritromicina

obstante el buen control del embarazo especialmente aquellos de alto riesgo; junto con algunas intervenciones como el adecuado diagnóstico y tratamiento de la infección urinaria, de la vaginosis bacteriana, de la corioammionitis, y en general de cualquier infección materna; podría reducir el parto prematuro ${ }^{8,9}$. Se ha publicado que la suplementación materna con antioxidantes, como la vitamina $\mathrm{C}$ y $\mathrm{E}$ reducen la ocurrencia de preeclampsia; de tal forma que podrían reducir la incidencia de parto prematuro?

\section{Administración de Corticoides Antenatales}

El uso de esteroides antenatales en madres con riesgo de parto prematuro ha reducido la incidencia de muertes neonatales y distrés respiratorio en aproximadamente un $50 \%{ }^{10}$. Sin embargo, esta medida junto con la administración de surfactante no ha logrado reducir la incidencia de DBP, aunque si su severidad ${ }^{1,2,10}$. Estudios con administración de TRH (hormona tiroestimulante) tampoco han logrado reducir esta afección ${ }^{11}$.

\section{Estrategias de Prevención Postnatales (tabla 1)}

\section{Probadas}

1. Nutrición Adecuada

Mantener una adecuada nutrición en los RN de muy bajo peso desde el nacimiento es de primordial importancia para tratar de prevenir o disminuir la DBP. Diversos nutrientes son indispensables para una adecuada función pulmonar y para el proceso de reparación. Una de las sustancias que se ha administrado es el Inositol, que es un precursor para la síntesis de surfactante pulmonar, el cual se ha reportado deficitario en los prematuros. Dos estudios realizados por Hallman y cols, hace más de 15 años demostraron que los $\mathrm{RN}$ prematuros suplementados con Inositol presentaban una significativa menor incidencia de DBP a los 28 días de vida ${ }^{12,13}$. Sin embargo, en el segundo ensayo clínico de este autor, el aumento de sobrevivientes sin DBP, no fue significativo entre aquellos RN que habían recibido surfactante exógeno; sugiriendo que la administración de Inositol no potenciaría el efecto del surfactante en los $\mathrm{RN}$ a riesgo de $\mathrm{DBP}^{13}$. Actualmente, la terapia con Inositol no es parte del tratamiento estándar de los $\mathrm{RN}$ de muy bajo peso.

La vitamina A participa en la regulación del crecimiento del desarrollo pulmonar y en la mantención y reparación del epitelio respiratorio después de una injuria ${ }^{14,15}$. El mayor paso de vitamina $\mathrm{A}$ hacia el feto ocurre en el último trimestre del embarazo; por lo cual los $\mathrm{RN}$ menores a 28-30 semanas de gestación nacen sin los depósitos adecuados de vitamina $\mathrm{A}^{15}$. Varios estudios han demostrado que la suplementación del RN de muy bajo peso, con vitamina $\mathrm{A}$ en dosis de $5000 \mathrm{U}$ IM tres veces por semana durante el primer mes de vida disminuye significativamente la incidencia de DBP (55\% versus 62\%) sin presentar alteraciones en el desarrollo neurosensorial ${ }^{15,16}$. El metaanálisis de 7 estudios demostró una pequeña, pero significativa reducción del resultado combinado muerte o DBP a los 28 días y 36 semanas postconcepcional ${ }^{17}$. Curiosamente a pesar del efecto beneficioso comprobado con la administración de vitamina A, muy pocas Unidades de 
Neonatología de Estados Unidos la utilizan ${ }^{18}$ y prácticamente ninguna de Sudamérica ${ }^{19}$.

También se ha utilizado la vitamina E como antioxidante en la prevención de la DBP, pero sin resultados efectivos ${ }^{20}$. La revisión sistemática de diversos estudios no demuestra reducción en la incidencia de DBP, ni tampoco de otras patologías respiratorias ${ }^{20}$.

\section{Administración de Cafeina}

Las metilxantinas entre las cuales están la aminofilina y cafeína son frecuentemente utilizadas en las unidades de neonatología para la prevención o tratamiento de los episodios de apnea en los prematuros ${ }^{21}$. Sin embargo, poco o nada se conocía de los efectos a largo plazo con esta terapia; hasta el estudio recientemente publicado por Schmidt y cols ${ }^{22}$. En dicho ensayo clínico en el cual se enrolaron 2006 neonatos con peso nacimiento entre 500 y $1250 \mathrm{~g}$; fueron randomizados a recibir cafeína o placebo dentro de los primeros 10 días de vida. El objetivo primario de este estudio fue determinar los efectos neurosensoriales a los 18-21 meses de edad corregida. El grupo tratado con cafeína demostró una mejoría significativa en su desarrollo neurosensorial ${ }^{22}$. Paralelamente, cuando se analizó la dependencia de oxígeno a las 36 semanas postconcepcional, se observó una significativa reducción en la incidencia de DBP en el grupo tratado con cafeína, postulando los autores que se debería a la menor duración de la ventilación mecánica en los niños tratados con cafeína, y también al efecto diurético y antiinflamatorio de este fármaco ${ }^{22}$.

Actualmente se considera que la cafeína, podría ser una droga efectiva en la prevención de la DBP en los RN de muy bajo peso ${ }^{23}$.

\section{Administración de Corticoides}

El uso de corticoides como prevención de la DBP es posiblemente la intervención más controversial de esta patología. Niveles bajos de cortisol plasmático en $\mathrm{RN}$ de muy bajo peso en los primeros días de vida, favorecerían una exagerada respuesta inflamatoria que podría contribuir al daño pulmonar y secundariamente al desarrollo de DBP ${ }^{24,25}$. Por ser los corticoides potentes antiinflamatorios, numerosos estudios en la década de los 90 , se efectuaron con la finalidad de prevenir o disminuir la $\mathrm{DBP}^{26-29}$. Estos ensayos clínicos postnatales que utilizaron fundamentalmente dexametasona se pueden agrupar, según la edad de inicio del tratamiento, en precoz (antes de los 4 días de vida), moderadamente precoz o intermedio ( 7 a 14 días) y tardío (después de los 21 días). Estos estudios demostraron mejoría en la función pulmonar, facilitan la extubación del RN y disminución de la $\mathrm{DBP}^{27,28}$. El metaanálisis de estos, demuestra una significativa reducción de la DBP a los 28 días de vida y 36 semanas postconcepcionales; pero con efectos adversos serios en el crecimiento craneano, y en el desarrollo pulmonar y neurosensorial ${ }^{27,28}$. Grier y Halliday, han calculado que por cada 100 niños que reciben corticoides en forma precoz, la DBP se puede prevenir en 10, a expensas de 6 niños con hemorragia gastrointestinal, 12 con parálisis cerebral y 14 con examen neurológico anormal durante el seguimiento ${ }^{29}$. También se han descrito otros efectos adversos con el uso de esteroides, como hiperglicemia, proteólisis, hipertensión arterial, miocardiopatía hipertrófica, disminución del crecimiento somático y pulmonar, supresión adrenal, aumento de infecciones y osteopenia ${ }^{30}$. Estos efectos adversos se han observado en los estudios que han utilizado dosis elevada de dexametasona y por tiempos prolongados, por lo tanto, aún persisten interrogantes acerca de sus beneficios al administrarlo por períodos más cortos y en dosis menores. Actualmente, en algunas unidades de neonatología se recomienda utilizarlos, previo consentimiento informado de los padres, por períodos cortos (3 a 7 días) en RN después de la tercera semana de vida, o en niños con DBP que permanecen conectados a ventilación mecánica, para facilitar el retiro del ventilador ${ }^{31,32}$. También se han utilizado corticoides por vía inhalatoria por tener menos efectos sistémicos colaterales; pero con menores beneficios a nivel pulmonar, que los corticoides por vía sistémica ${ }^{33}$.

Por los efectos dañinos de los corticoides en el desarrollo neurológico de los neonatos, la Academia Americana de Pediatría, no recomienda la administración de corticoides postnatales en $\mathrm{RN}$ a riesgo de $\mathrm{DBP}^{31}$. 


\section{No Probadas}

\section{Manejo Conservador y Cuidadoso en las Maniobras de Reanimación}

Los RN menores de $1500 \mathrm{~g}$ requieren al nacer más frecuentemente que los de término o cercano al término de algún tipo de maniobras de reanimación; especialmente ventilación a presión positiva (VPP) con bolsa autoinflable por no respirar en forma adecuada y/o rítmi$\mathrm{ca}^{34,35}$. Estos neonatos que no tienen una transición satisfactoria en su respiración en los primeros segundos o minutos de vida, deben recibir una asistencia ventilatoria lo más gentil posible para evitar injuriar el pulmón inmaduro. Se ha demostrado en experimentación animal que bastan 8 a 10 ventilaciones con excesivo volumen corriente para producir daño al pulmón $^{36}$. Para evitar el exceso de volumen corriente, el ideal es proporcionar VPP con bolsa autoinflable con un manómetro de presión. Durante las primeras 10 ventilaciones con bolsa autoinflabe debe usarse una presión inspiratoria de alrededor de $30 \mathrm{~cm} \mathrm{H}_{2} \mathrm{O}$, para descender posteriormente a $20 \mathrm{~cm} \mathrm{H}_{2} \mathrm{O}^{34}$. También sería importante proporcionar desde las primeras VPP, una presión positiva al final de la expiración (Peep), para obtener lo antes posible una adecuada capacidad funcional residual, evitando el colapso alveolar al final de la expiración ${ }^{37}$.

La recomendación actual para reanimar un recién nacido que no respira es ventilarlo con $100 \%$ de oxígeno ${ }^{34}$. Sin embargo, el metaanálisis de estudios randomizados controlados que han comparado la reanimación neonatal con $100 \%$ de $\mathrm{O}_{2}$ versus $21 \%$ de $\mathrm{O}_{2}$ han sugerido un aumento de la mortalidad en los RN reanimados con $100 \%$ de $\mathrm{O}_{2}{ }^{38}$. Además en varias oportunidades en $\mathrm{RN}$ con adecuada ventilación espontánea y frecuencia cardíaca mayor de 100 por minuto, pero con cianosis central; que a veces es subjetiva; se usa indiscriminadamente oxígeno a flujo libre al $100 \%$, generalmente seco y frío por varios minutos ${ }^{39}$, favoreciendo el estrés oxidativo en los pacientes, al acumularse radicales libres que pueden dañar el pulmón y otros tejidos del $\mathrm{RN}^{40}$.

El ideal sería tener en toda sala de atención de RN inmediato, un mezclador de aire y oxígeno, para regular la fracción inspirada de oxígeno, junto con un oxímetro de pulso, para medir continuamente la saturación de oxígeno y determinar en forma más precisa la frecuencia cardíaca del niño ${ }^{37}$. Paralelamente, si se midiera el volumen corriente mientras se proporciona VPP en los RN que requieren ventilación en los primeros minutos de vida, sería un importante avance en evitar la sobredistensión pulmonar y también la hipocapnia, que puede producir daño cerebral ${ }^{37}$.

Relativamente poca importancia se le ha dado a las maniobras de reanimación en los primeros segundos o minutos de vida, especialmente en los RN de muy bajo peso, y el posible daño pulmonar; siendo probable que altas concentraciones de oxígeno y de presiones inspiratorias condicionen una injuria pulmonar a futuro.

\section{Tratamiento con Surfactante}

La administración de surfactante exógeno, cambio la evolución del distrés respiratorio, facilitando el manejo del recién nacido con SDR, al evitar la ventilación mecánica y/o reducir significativamente su duración y complicaciones ${ }^{41}$. La terapia con surfactante ha disminuido la mortalidad neonatal, el baro-volutrauma y la asistencia respiratoria del $\mathrm{RN}$, pero no ha reducido la incidencia de $\mathrm{DBP}^{3,41}$; posiblemente debido al aumento en la sobrevivencia de los neonatos más inmaduros, que son los más expuestos a desarrollar esta patología $a^{4,42}$. No obstante cuando el resultado final es la combinación de DBP y muerte, los niños que han recibido surfactante resultan claramente beneficia$\operatorname{dos}^{41,42}$.

\section{Oxígenoterapia}

Por estar claramente demostrado el efecto tóxico del oxígeno a nivel pulmonar, debe utilizarse con indicaciones precisas y por el menor tiempo posible. Hasta hoy se desconoce cual sería el nivel de saturometría ideal para un $\mathrm{RN}$, pero hay consenso mayoritario, de acuerdo a los resultados de estudios recientes, que saturaciones sobre $95 \%$ producen más daño pulmonar ${ }^{43,44}$. Es muy posible que en la etapa aguda del distrés respiratorio, mantener saturaciones entre 88 y $95 \%$ y $\mathrm{PaO}_{2}$ entre 50 y 70 mmHg sean adecuados. Se requieren nuevos estudios colaborativos y randomizados para de- 
terminar cuales serían los rangos óptimos de saturación de oxígeno, especialmente en los $\mathrm{RN}$ prematuros menores de 28 a 30 semanas de edad gestacional en las primeras semanas de vida.

\section{Uso Precoz de CPAP Nasal}

La aplicación de presión positiva continua en vía aérea (CPAP) precozmente después del nacimiento, en vez de la intubación endotraqueal y conexión a ventilación mecánica se ha asociado con menor incidencia de $\mathrm{DBP}^{45-47}$. También se ha observado que el uso rutinario de CPAP en RN mayores de 25 semanas de edad gestacional, ha mejorado los resultados para los $\mathrm{RN}$, al aumentar la experiencia de los neonatólogos ${ }^{48,49}$.

Al utilizar CPAP precoz se ha logrado evitar la ventilación mecánica en aproximadamente un tercio de los RN con edad gestacional de 25 semanas o menos y cerca de un $80 \%$ en recién nacidos con 28 o más semanas de edad gestacional $^{48}$. En la Universidad de Columbia EE.UU. Wang y col, desde hace 25 a 30 años utilizan CPAP nasal precoz, como estrategia ventilatoria primaria en los $\mathrm{RN}$, reportando en forma sostenida incidencias bajas de DBP en comparación con otros centros neonatales de similar complejidad ${ }^{45,46}$. Sin embargo, son reportes de la experiencia local, que no han sido comparados prospectivamente con otros métodos de asistencia respiratoria. Situación similar ocurre con diversos estudios, que comparan el uso precoz de CPAP nasal versus el grupo control histórico, de años anteriores; efectuados en el mismo centro, con resultados alentadores respecto del beneficio del uso de CPAP precoz en la disminución de $\mathrm{DBP}^{48,49}$. En la red Sudamericana de Neonatología (Neocosur) en un período de 6 años (2001-2007) se detectó también un descenso significativo de los niños dependientes de oxígeno a las 36 semanas postconcepcionales ${ }^{19}$, posiblemente debido al significativo aumento del uso de CPAP en igual período de tiempo en las diferentes unidades (21\% a $48 \%)$. No obstante, al no ser estudios prospectivos, randomizados, persisten las dudas o interrogantes acerca de la real utilidad de esta intervención en la disminución de la DBP. Recientemente un estudio prospectivo rando- mizado y multicéntrico, que utilizó desde el nacimiento CPAP nasal versus intubación y ventilación mecánica, no demostró diferencias en la incidencia de DBP a las 36 semanas postconcepcionales, observándose como complicación un mayor porcentaje de neumotórax en el grupo tratado con CPAP (9\% versus $3 \%)^{50}$.

La práctica clínica de utilizar CPAP nasal precozmente en $\mathrm{RN}$ de muy bajo peso, parece una intervención promisoria, pero aún faltan más estudios prospectivos, multicéntricos y randomizados para determinar si realmente disminuye la DBP.

\section{Soporte Ventilatorio Mecánico}

En RN muy inmaduros generalmente menores de $1000 \mathrm{~g}$, la asistencia ventilatoria mecánica es relativamente frecuente, debido a falta de esfuerzo respiratorio, o bien a episodios de apnea ${ }^{1,5}$. En estos casos debe tratar de utilizarse la ventilación más fisiológica para el $\mathrm{RN}$, manteniendo volúmenes corrientes entre 4 y 5 $\mathrm{ml} / \mathrm{kg}$ con las menores presiones inspiratorias y concentraciones de oxígeno posible, para mantener saturaciones entre 88 y $95 \%{ }^{1,51}$. Idealmente debe evitarse en todo momento presiones inspiratorias elevadas, que pueden conducir a volúmenes corrientes elevados y producir escapes aéreos (volutrauma) ${ }^{52}$. Por tal motivo, en los últimos años se ha utilizado la ventilación gentil o mínima, que permite niveles de $\mathrm{PaCO}_{2}$ entre 50 y $60 \mathrm{mmHg}$, la denominada "hipercapnia permisiva", que podría evitar o minimizar el daño pulmonar $^{53}$. Sin embargo, el metaanálisis de los estudios con hipercapnia permisiva, no mostró un efecto protector en disminuir la DBP, sugiriendo este resultado efectuar nuevos estudios clínicos ${ }^{54}$. Otro de los nuevos métodos de ventilación mecánica que han sido evaluados para minimizar la injuria pulmonar, es la ventilación sincronizada o gatillada por el paciente ${ }^{55}$. Lamentablemente los estudios con este tipo de ventilación no han mostrado reducción de la DBP, sólo han permitido disminución en la duración de la ventilación mecánica ${ }^{1,55}$.

En los últimos 20 años en la era pre y post surfactante, se han realizado numerosos ensayos clínicos comparando la ventilación convencional, con la ventilación de alta frecuencia (VAF) en RN de muy bajo peso con distrés 
respiratorio, con resultados contradictorios ${ }^{56-58}$. Los dos estudios con mayor número de casos enrolados, con corticoides antenatales y uso de surfactante exógeno demostraron diferentes resultados ${ }^{56,57}$. En uno de los estudios con pacientes con distrés respiratorio moderado o severo y que utilizó sólo un tipo de VAF, mostró significativa menor incidencia de DBP, en los niños tratados con VAF, y se lograron extubar una semana antes que los niños tratados con ventilación sincronizada ${ }^{56}$. El otro estudio con casi 800 neonatos, no demostró ninguna diferencia entre los niños tratados con ventilación convencional o VAF, reportando igual incidencia de DBP, escapes aéreos, hemorragia intracraneana y leucomalasia periventricular ${ }^{57}$.

El modo ideal de ventilación del RN, especialmente prematuro, hasta el momento no se ha encontrado; pero los actuales conocimientos indican que los prematuros que requieren soporte ventilatorio mecánico, deben mantenerse el menor tiempo posible y ser manejados con volúmenes corrientes pequeños y las menores presiones inspiratorias y concentraciones de oxígeno posible, evitando la hipocapnia y el volutrauma.

\section{Cierre Precoz del Ductus Arterioso y Restricción de Líquidos}

El ductus arterioso persistente se ha asociado a $\mathrm{DBP}^{2,5,59}$, y el exceso de líquido en los primeros días de la vida favorece la mantención o reapertura del ductus arterioso ${ }^{60}$. Por tal motivo, la restricción de líquidos y el cierre precoz del ductus utilizando indometacina o ibuprofeno son prácticas clínicas habituales en los $\mathrm{RN}$ de muy bajo peso. Sin embargo, diversos estudios han fracasado en demostrar, que el cierre precoz del ductus, por la administración profiláctica de indometacina en comparación con placebo, disminuyen la incidencia de la $\mathrm{DBP}^{61,62}$. Incluso en el análisis efectuado por Schmidt y cols ${ }^{61}$, se observó un aumento en la incidencia de DBP en aquellos recién nacidos tratados con indometacina profiláctica, que no presentaron ductus. Este resultado se explicaría, por los efectos colaterales producidos por la indometacina como son, la disminución de la diuresis, que produce retención de líquidos, y el aumento de los requerimientos de oxígeno durante la primera semana de vida ${ }^{61}$. Paralelamente, la no disminución de la DBP por el cierre precoz del ductus, se podría deber al hecho, que un número significativo de RN controles también recibieron indometacina en algún momento de su evolución, cuando el ductus se hizo sintomático. También está la observación que la DBP tiene relación con la duración del ductus arterioso, y no sólo con su presencia ${ }^{63}$.

De acuerdo a estos resultados, algunos autores han postulado que el ductus por sí solo, no tendría efecto en la patogénesis de la DBP; sino que sería un marcador de riesgo ${ }^{61}$. No obstante faltan nuevos estudios para determinar con mayor exactitud el rol del ductus arterioso en la DBP.

\section{Antioxidantes}

La inmadurez de los sistemas antioxidantes de defensa que incluyen principalmente la superóxido-dismutasa, la catalasa y la glutationperoxidasa, exponen a los RN prematuros, especialmente de extremo bajo peso, al estrés oxidativo producido por los radicales libres de oxígeno ${ }^{64}$. Diversos estudios en experimentación animal han demostrado que la administración de antioxidantes exógenos reduce el daño pulmonar. Sin embargo, los estudios efectuados en humanos han tenido resultados desalentadores. La N-acetylcysteina, que esta muy relacionada con la glutatión peroxidasa, se evaluó su efecto endovenoso en $391 \mathrm{RN}$ de extremo bajo peso, quienes estaban en ventilación mecánica o CPAP a las 36 horas de vida ${ }^{65}$. Este antioxidante no disminuyó el resultado combinado de muerte o DBP, y tampoco se observaron diferencias en la función pulmonar entre los grupos. Otro ensayo randomizado, doble ciego que evaluó la aplicación de superóxido-dismutasa o placebo en $302 \mathrm{RN}$ prematuro con peso nacimiento entre 600 y $1200 \mathrm{~g}$, con distrés respiratorio, que recibieron surfactante exógeno y estaban en ventilación mecánica, no demostró ningún efecto en la incidencia de DBP a los 28 días de vida y/o las 36 semanas de edad postconcepcional ${ }^{66}$. No obstante, al año de edad corregida los niños tratados con superóxidodismutasa presentaron un número significativamente menor de episodios de sibilancias, de visitas a la unidad de emergencia, de hospitali- 
zaciones y de uso de broncodilatadores y/o corticoides ${ }^{66}$. Estos datos sugieren que la terapia con antioxidantes exógeno podría atenuar o disminuir las secuelas pulmonares a largo plazo; para lo cual es necesario efectuar nuevos estudios.

\section{8. Óxido Nitrico Inhalado}

En los últimos años se ha utilizado la administración de óxido nítrico inhalado (ONi), como otra de las posibles intervenciones en la prevención de la DBP. Los mecanismos por los cuales este gas podría disminuir la DBP son varios, entre los cuales estarían; acción antiinflamatoria, mejoría de la relación ventilación/ perfusión, disminución de la resistencia vascular pulmonar; aumento de la broncodilatación, promoción de la remodelación pulmonar en respuesta a la injuria y normalización de la función del surfactante ${ }^{67-69}$.

Los diferentes ensayos clínicos que han utilizado ONi en $\mathrm{RN}$ prematuros han proporcionado resultados contradictorios ${ }^{67-69}$. El estudio de Schreiber y cols ${ }^{67}$, randomizó en un solo centro a $207 \mathrm{RN}$ ventilados con ONi o placebo, antes de las 72 horas de vida. Los RN tenían un peso de nacimiento menor de $2000 \mathrm{~g}$ y una edad gestacional menor de 34 semanas. Los RN que recibieron $\mathrm{ONi}$ presentaron significativamente menor mortalidad o DBP, que el grupo de RN que recibieron placebo. Uno de los últimos estudios publicados es el de Ballard y cols ${ }^{68}$, que enroló a $582 \mathrm{RN}$, con peso de nacimiento entre 500-1250 g en ventilación mecánica y que iniciaron ONi entre el 7 y 14 días de vida. La sobrevivencia sin DBP aumentó en el grupo tratado versus el grupo control (43,9\% versus $38,6 \%, \mathrm{p}<0,04)$. Por el contrario el estudio multicéntrico, randomizado de Kinsella y cols ${ }^{69}$, en 793 recién nacidos menores de 34 semanas de edad gestacional, con un peso de nacimiento entre 500 y 1250 g, que inició el ONi a las 48 horas de vida y lo continuó hasta los 21 días; en aquellos niños que permanecieron en ventilación mecánica y recibieron $\mathrm{ONi}$ en dosis de 5 ppm, no se demostró disminución en la incidencia global de DBP. Sólo se reportó disminución de la DBP en el grupo de niños con peso mayor a $1000 \mathrm{~g}$, que son los neonatos que tienen menor incidencia de DBP. Sin embargo, este estudio redujo el riesgo de daño cerebral en los niños tratados con ONi (17,5\% versus $23,9 \%)$, demostrando un efecto neuroprotector ${ }^{69}$. Recientemente se ha conocido en forma de resumen el estudio Europeo del efecto de ONi en el desarrollo de enfermedad pulmonar crónica en RN prematuros (European Nitric Oxide: EUNO estudio ${ }^{70}$. En este ensayo clínico se reclutaron $800 \mathrm{RN}$ de 36 centros de 9 países de Europa. Todos los neonatos tenían menos de 29 semanas de edad gestacional (promedio 26,5 sem), y un peso promedio de $835 \mathrm{~g}$. El $90 \%$ de los niños recibieron esteroides antenatales, e igual porcentaje fue intubado antes del ingreso al estudio. Todos los $\mathrm{RN}$ recibieron surfactante dentro de las primeras 12 horas de vida. Este estudio deliberadamente excluyó prematuros con insuficiencia respiratoria severa $(\mathrm{RN}$ que requerían más de $50 \%$ de oxígeno para mantener una saturación sobre $85 \%$, luego de 2 horas de haber recibido surfactante). Aquellos RN asignados al grupo ONi y que recibieron $5 \mathrm{ppm}$ por 7 a 21 días, tuvieron igual sobrevivencia, que el grupo placebo ( $85,8 \%$ versus $89,5 \%)$; e igual dependencia de oxígeno a las 36 semanas postconcepcionales $(65,3 \%$ versus $65,5 \%)$. Este ensayo clínico, que es el de mayor número de pacientes enrolados, claramente demuestra que el uso profiláctico de ONi no aumenta la sobrevida, ni reduce la incidencia de DBP en RN menores de 29 semanas de edad gestacional y con distrés respiratorio leve a moderado ${ }^{70}$. Por lo tanto, esta terapia que es posiblemente la de mayor costo en neonatología, esta aún en etapa experimental, debiendo esperarse nuevos estudios que determinen el real beneficio y dilucidar algunas interrogantes que aún persisten como, el inicio y la población de niños más adecuados; la dosis óptima y la duración del tratamiento con $\mathrm{ONi}$.

\section{Administración de Eritromicina}

Diversos estudios observacionales han demostrado la asociación entre colonización de la vía aérea con Ureaplasma Urealyticum y mayor riesgo de $\mathrm{DBP}^{71}$. Sin embargo, dos estudios randomizados que utilizaron Eritromicina, fracasaron en demostrar algún efecto en reducir la incidencia de DBP a las 36 semanas postconcepcionales $^{72,73}$. La pérdida de efecto be- 
neficioso al usar Eritromicina se debería a la falla en la eliminación del U. Urealyticum del pulmón del RN, situación que aún no ha sido suficientemente probada ${ }^{74}$. También se especula que la terapia postnatal con Eritromicina contra el U. Urealyticum sería tardía, al producirse la infección in útero, con la consiguiente inflamación e injuria del pulmón ${ }^{74}$. Se requieren mayores estudios evaluando el momento más óptimo en la administración de la Eritromicina como medida de prevención de la DBP.

\section{Tratamiento}

Si las diferentes intervenciones preventivas anteriormente nombradas, no pudieron impedir el desarrollo de DBP, se pueden efectuar algunas medidas terapéuticas, teniendo como objetivo mantener un adecuado intercambio gaseoso, mientras se evita la progresión de la enfermedad.

\section{Oxígenoterapia}

El oxígeno es la droga más común utilizada en las Unidades de Neonatología, y es parte muy importante en el soporte respiratorio neonatal ${ }^{75,76}$. Con el oxígeno se da la paradoja, que sus elevadas concentraciones son uno de los factores de riesgo más importantes en la etiopatogenia de la DBP; pero a la vez es parte del tratamiento del paciente con esta patología. La técnica más utilizada para el monitoreo continuo y no invasivo de la oxígenoterapia es la oximetría de pulso ${ }^{75,76}$.

No existe suficiente información para recomendar un rango exacto de saturación de oxígeno o $\mathrm{PaO}_{2}$ en $\mathrm{RN}$ que estén cursando con DBP, que permita evitar la toxicidad potencial por oxígeno, mientras se provee adecuada entrega de oxígeno a los tejidos ${ }^{76}$. Por tal motivo, hay una gran variabilidad en la práctica clínica, respecto del nivel de saturación de oxígeno adecuado para estos niños ${ }^{75,76}$. No obstante, tal como se mencionó anteriormente hay bastante evidencia para afirmar, que saturaciones de oxígeno mayores de $95 \%$ y $\mathrm{PaO}_{2}$ mayores de $80 \mathrm{mmHg}$ pueden asociarse con un peor pronóstico respiratorio ${ }^{43,44}$. Por lo tanto, es recomendable mantener la saturación de oxígeno entre 90 y $95 \%$, y/o la $\mathrm{PaO}_{2}$ entre 50 y 70 $\mathrm{mmHg}$ con la finalidad, de minimizar los efectos deletéreos de la hipoxia e hiperoxia. Para aquellos niños con DBP que tienen una hipertensión pulmonar, deberían mantenerse con saturaciones levemente mayores, entre $92 \%$ y 96\%. Estos niveles adecuados de oxigenación deben mantenerse en los niños con DBP en todas las circunstancias, como son al alimentarse, durante el sueño, o durante cualquier otra actividad.

Los intentos para interrumpir el oxígeno suplementario deben efectuarse durante las horas de vigilia, con monitoreo permanente de la oxigenación, a través de la oximetría de pulso, para asegurarse que el paciente mantiene saturaciones sobre $95 \%$ en diversas circunstancias. Es importante considerar que factores como el hematocrito y el flujo sanguíneo estén dentro de los rangos normales, a fin de favorecer una mejor oxigenación tisular. La optimización de estos factores es crítica y pueden permitir una oxigenación adecuada con mínima administración de oxígeno suplementario ${ }^{75,76}$.

En el pasado, la administración crónica de oxígeno obligaba a hospitalizaciones prolongadas; no obstante en los últimos años se han descrito buenos resultados con el tratamiento domiciliario de estos niños ${ }^{77}$.

\section{Diuréticos}

El empleo de diuréticos disminuye la resistencia pulmonar y mejoran la distensibilidad pulmonar en niños con DBP. Sin embargo, no cambian el curso de la enfermedad, aunque son útiles en descompensaciones asociadas a un aumento del edema pulmonar ${ }^{78-80}$.

El tratamiento con diuréticos como la furosemida se utiliza por cortos períodos de tiempo (2 a 3 días), y luego se deja un período de descanso. Raramente se utiliza por tiempos prolongados puesto que el uso crónico, especialmente de la furosemida puede producir graves efectos secundarios, como trastornos hidroelectroliticos: hipocalemia, hiponatremia e hipocronemia, y alcalosis metabólica. También puede producir hipercalciuria asociada a nefrocalcinosis. Por lo tanto, al ser necesario prescribirlos por períodos prolongados, deben administrarse en días alternos ( 1 a $2 \mathrm{mg} / \mathrm{kg}$ cada 48 
hr). Para evitar los efectos colaterales de la furosemida se han utilizado por vía inhalatoria, con resultados contradictorios ${ }^{80}$. Igualmente se pueden utilizar otros diuréticos como la hidroclorotiazida y la espironolactona, que tienen menos efectos secundarios, pero al mismo tiempo menor efectividad clínica. Tres revisiones sistemáticas que evaluaron el efecto de la furosemida e hidroclorotiazida en la prevención o tratamiento de DBP, no mostraron ningún efecto beneficioso a largo plazo ${ }^{78-80}$. Cualquiera sea el diurético utilizado deben controlarse periódicamente los electrolitos plasmáticos.

\section{Broncodilatadores}

Los niños con DBP tienen frecuentemente obstrucción bronquial por hipertrofia del músculo liso de la vía aérea ${ }^{81}$. Para el tratamiento de la DBP se han utilizado diversos broncodilatadores por vía inhalatoria y sistémica ${ }^{82}$. Entre los broncoditatadores inhalados que se han usado, están los $\beta 2$ agonistas como salbutamol, isoproterenol, orciprenalina, terbutalina $\mathrm{y}$ agentes anticolinérgicos como atropina $\mathrm{y}$ bromuro de ipratropio, que mejoran la función pulmonar y revierten parcialmente y en forma transitoria la obstrucción bronquial ${ }^{82-84}$. No existe evidencia de que el empleo prolongado de los broncodilatadores cambie el curso de la $\mathrm{DBP}^{83}$. La mayoría de estos fármacos son de vida media corta y también tienen una acción cardiovascular produciendo taquicardia, hipertensión arterial y ocasionalmente arritmias ${ }^{83,84}$. Por tal motivo, su uso debe limitarse en casos de exacerbación de la obstrucción bronquial. El método de administración de los inhaladores es muy importante, habiéndose demostrado un mejor efecto a través de una aerocámara o espaciador, en vez de la nebulización ${ }^{81,83}$. Si los RN se mantienen en ventilación mecánica se pueden administrar los broncodilatadores en la rama inspiratoria del respirador. Las metilxantinas como, la aminofilina y la cafeína, muy útiles en la prevención y tratamiento de la apnea del prematuro, también tienen una acción broncodilatadora, disminuyendo la resistencia de las vías aéreas, junto con otros potenciales beneficios, como la estimulación del centro respiratorio, aumento de la contractibilidad diafragmática y además un efecto diurético ${ }^{21,22,85}$.

\section{Manejo de la Hidratación y Nutrición}

El recién nacido con DBP tolera mal los líquidos, los cuales se acumulan en el tejido intersticial del pulmón, produciendo edema pulmonar, con deterioro de la relación ventilación/perfusión, pudiendo conducir a hipoxemia e hipercapnia. Por tal motivo, la ingesta de líquidos debe restringirse al máximo, pero manteniendo un volumen suficiente, para aportar las calorías necesarias para sus requerimientos metabólicos y de crecimiento. La nutrición adecuada es fundamental en los pacientes con DBP, para garantizar un adecuado crecimiento somático y del pulmón. Leches con alto contenido calórico y de calcio y fósforo, pueden ser útiles para proporcionar los requerimientos calóricos y proteicos necesarios, en un menor volumen de líquido.

\section{Corticoides}

Por la importancia que el proceso inflamatorio tiene en la patogenia de la DBP los corticoides como droga antiinflamatoria tienen un rol durante la fase aguda de la enfermedad ${ }^{24,30}$. Diversos reportes clínicos han demostrado un efecto beneficioso de los corticoides a corto plazo a nivel de la función pulmonar, facilitando el retiro de los niños del ventilador y disminución de los días de oxígeno suplementario ${ }^{27-29}$. Sin embargo, por sus efectos secundarios a largo plazo, especialmente a nivel del sistema nervioso central, su indicación actualmente se limita al RN con más de 14 días de vida, que no se logra extubar, y que está con parámetros ventilatorios elevados. Su dosis aún no está bien definida, pero en general se utilizan $0,20-0,25 \mathrm{mg} / \mathrm{kg} /$ dosis cada 8 horas por dos días y $0,1 \mathrm{mg} / \mathrm{kg}$ / dosis por otros dos días. Si la extubación se logra antes de completar el tratamiento se suspenden. Es preferible solicitar consentimiento informado antes de utilizar este fármaco.

También se han utilizado corticoides inhalatorios como una terapia alternativa antiinflamatoria, con menor efecto que los por vía sistémica, pero sin los riesgos asociados descritos con la administración sistémica ${ }^{33,86}$. Se usan por períodos variables, entre una a cuatro semanas; con lo cual generalmente se logra una extubación exitosa ${ }^{33}$. Los corticoides más utilizados son la beclometasona (50 ug cada 8 
horas) y budesonide (100 ug cada 12 horas). No obstante, se requieren mayores estudios para determinar el tipo de corticoide; las dosis y vía de administración; el tiempo mínimo necesario y conocer exactamente sus efectos neurológicos a largo plazo.

\section{Control de Infecciones}

Las infecciones sistémicas favorecen el desarrollo de la DBP, y agravan la ya existente; produciendo un retroceso del cuadro clínico ${ }^{5,71}$. Por lo tanto, es fundamental hacer todos los esfuerzos necesarios para evitar las infecciones nosocomiales o intrahospitalarias. Entre las medidas existentes para prevenir infecciones nosocomiales, están, el riguroso lavado de manos de todo el personal que atiende al $\mathrm{RN}$, siendo esta la medida más importante, uso de equipos estériles, evitar la multipunción de los pacientes, mantener indemnidad de la piel; restringir las intervenciones invasivas (intubación, cateterización, etc) y mantenerlas el menor tiempo posible, y el aislamiento de las personas con infecciones respiratorias. También es importante buscar signos y síntomas precoces de infección, a través de un cuidadoso examen clínico y exámenes de laboratorio; junto con cultivos periódicos de secreciones bronquiales. Frente a la sospecha clínica de infección sistémica debe iniciarse precozmente antibioterapia; pero si la evolución clínica y los exámenes de laboratorio a las 48 horas están normales, suspender los antibióticos. El sobretratamiento con antibióticos puede seleccionar cepas microbianas resistentes y más virulentas.

\section{Inmunización y Anticuerpos Monoclonales}

Los niños con DBP tienen mayor riesgo de infecciones respiratorias a repetición y mayor número de hospitalizaciones ${ }^{81,87}$. El agente etiológico que más frecuentemente afecta a estos niños es el virus respiratorio sincisial $(\mathrm{VRS})^{87}$. El mayor estudio con anticuerpo monoclonal humanizado (palivizumab), redujo la tasa de hospitalización por VRS en niños con DBP en un $4,9 \%$ (de $12,8 \%-7,9 \%)^{88}$. Esto significa que 20 niños con DBP deben recibir un curso de palivizumab, para prevenir una hospitalización por VRS.

La recomendación actual de la Academia
Americana de Pediatría es efectuar profilaxis con palivizumab o inmunoglobulina antiVRS, en niños menores de 2 años de edad con DBP, quienes han requerido tratamiento por su enfermedad pulmonar dentro de los 6 meses antes de la estación de $\operatorname{VRS}^{88}$.

\section{Conclusión}

A pesar de los avances de la medicina perinatal y neonatal de los últimos años, la DBP continua siendo una de las secuelas más frecuentes que afecta al RN de muy bajo peso. Desde la descripción inicial por Northway hace más de 40 años, nuevas terapias preventivas han emergido, habiéndose demostrado que la administración de vitamina A y cafeína disminuyen la incidencia de esta enfermedad. Otras intervenciones preventivas como la administración de antioxidantes y óxido nítrico inhalado están siendo estudiados. Los nuevos modos de ventilación mecánica como la ventilación sincronizada y la ventilación de alta frecuencia no han disminuido la incidencia de esta afección.

Faltan estudios para determinar con mayor exactitud el posible rol del cierre farmacológico temprano del ductus arterioso persistente y de la utilización de esteroides postnatales en la prevención de esta enfermedad.

\section{Referencias}

1.- Kinsella JO, Greenough A, Abman SH: Bronchopulmonary Dysplasia. Lancet 2006; 367: 1421-31.

2.- Rojas M, González A, Bancalari E, et al: Changing trends in the epidemiology and pathogenesis of chronic lung disease. J Pediatr 1995; 126: 605-10.

3.- Walsh MC, Szefler S, Davis J, et al: Summary proceedings from the bronchopulmonary dysplasia group. Pediatrics 2006; 117: S52-S56.

4.- Fanaroff AA, Stoll BJ, Wright LL, et al: Trends in neonatal morbidity and mortality for very low birthweight infants. Am J Obstet Gynecol 2007; 196: 147.e1-147.e8.

5.- Bancalari E, Claure N, Sosenko IRS: Bronchopulmonary dysplasia: changes in pathogenesis, epidemiology and definition. Semin Neonatol 2003; 8: $63-71$.

6.- Van Marter LJ: Progress in discovery and evaluation 
of treatments to prevent bronchopulmonary dysplasia. Biol Neonate 2006; 89: 303-12.

7.- Thomas $W$, Speer CP: Prevention and treatment of bronchopulmonary dysplasia: current status and future prospects. J Perinatol 2007; 27 (suppl 1): S26-S32.

8.- Goldenberg RL, Rouse DJ: Prevention of Premature Birth. N Engl J Med 1998; 339: 313-20.

9.- Chappell LC, Seed PT, Briley AL, et al: Effects of antioxidants in the occurrence of pre-eclampsia in women at increased risk: a randomized trial. Lancet 1999; 354: 810-6.

10.- Crowley PA: Antenatal corticosteroid therapy: a metaanalysis of the randomized trials, 1972 to 1994 . Am J Obstet Gynecol 1995; 173: 322-35.

11.- Crowther CA, Alfirevic Z, Haslam RR: Thyrotrophin releasing hormone added to corticosteroids for women at risk of preterm birth for preventing neonatal respiratory disease. Cochrane Database Syst Rev 2004; 2: CD000019.

12.- Hallman M, Jarvenpaa AL, Pohjavuori M: Respiratory distress síndrome and inositol supplementation in preterm infants. Arch Dis Child 1986; 16: 1076-83.

13.- Hallman M, Bry K, Норри K: Inositol supplementation in premature infants with respiratory distress syndrome. N Engl J Med 1992; 326: 1233-9.

14.- Takahashi Y, Miura T, Takahashi K: Vitamin A is involved in maintenance of epithelial cells on the bronchioles and cells in the alveoli of rats. J Nutr 1993; 123: 634-41.

15.- Shenai JP, Chytil F, Stahlman MT: Vitamin A status of neonates with bronchopulmonary dysplasia. Pediatr Res 1985; 19: 185-9.

16.- Tyson JE, Wright LL, Oh $Q$, et al: Vitamin A supplementation for extremely low birth weight infants. N Engl J Med 1999; 340: 1962-8.

17.- Darlow BA, Graham PJ: Vitamin A supplementation for preventing morbidity and mortality in very low birthweight infants. Cochrane Database Syst Rev 2002; 4: CD000501.

18.- Ambalavanan N, Kennedy K, Tyson J, Carlo W: Survey of vitamin A supplementation for extremely-low-birthweight infants: is clinical practice consistent with the evidence? J Pediatr 2004; 145: 304-7.

19.- Tapia JL, D’Apremont I, Bancalari A, et al: Resultados Perinatales en la Red Neocosur. Período 20012006. Libro Resumen V Congreso Iberoamericano de Neonatología-Siben 2008; $52 \mathrm{~A}$.

20.- Brion LP, Bell EF, Raghuveer TS: Vitamin E supplementation for prevention of morbidity and mortality in preterm infants. Cochrane Database Syst Rev 2003; 4: CD003665.

21.- Henderson-Smart DJ, Steer P: Methylxantine treatment for apnea in preterm infants. Cochrane Database
Syst Rev 2001; 3: CD000140.

22.- Schmidt B, Roberts RS, Davis P, et al: Long-term effects of caffeine therapy for apnea of prematurity. N Engl J Med 2007; 357: 1893-902.

23.- Schmidt B, Roberts R, Millar D, Kirpalani H: EvidenceBased Neonatal Drug Therapy for Prevention of Bronchopulmonary Dysplasia in Very-Low Weight Infants. Neonatology 2008; 93: 284-7.

24.- Watterberg KL, Scott SM, Backstrom C, et al: Links between early adrenal function and respiratory outcome in preterm infants: airway inflammation and patent ductus arteriosus. Pediatrics 2000;105:320-4.

25.- Watterberg KL, Gerdes JS, Gifford KL, Lin HM: Prophylaxis against early adrenal insufficiency to prevent chronic lung disease in premature infants. Pediatrics 1999; 104: 1258-63.

26.- Tapia JL, Ramírez R, Cifuentes J, et al: The effects of early dexamethasone administration on bronchopulmonary dysplasia in preterm infants with respiratory distress syndrome. J Pediatr 1998; 132: 48-52.

27.- Halliday HL, Ehrenkranz RA, Doyle $L W$ : Early postnatal ( $<96$ hours) corticosteroids for preventing chronic lung disease in preterm infants. Cochrane Database Syst Rev 2003; 1: CD001146.

28.- Halliday HL, Ehrenkranz RA, Doyle LW: Moderately early (7-14 days) postnatal corticosteroids for preventing chronic lung disease in preterm infants. Cochrane Database Syst Rev 2003; 1: CD001144.

29.- Grier DC, Halliday HL: Corticosteroids in the prevention and management of bronchopulmonary dysplasia. Semin Neonatal 2003; 8: 83-91.

30.- Yeh TF, Torre JA, Rastogi A, Anyebuno MA, Pildes $R$ : Early postnatal dexamethasone therapy in pramature infants with severe respiratory distress syndrome: a double-blind, controlled study. J Pediatr 1990; 117: 273-82.

31.- American Academy of Pediatrics, Canadian Paediatric Society: Postnatal corticosteroid to treat or prevent chronic lung disease in preterm infants. Pediatrics 2002; 109: 330-8.

32.- Thomas $W$, Speer CP: Nonventilatory Strategies for Prevention and Treatment of Bronchopulmonary Dysplasia-What Is the Evidence? Neonatology 2008; 94: 150-9.

33.- Cole CH, Colton T, Shah BL, et al: Early inhaled glucocorticoid therapy to prevent bronchopulmonary dysplasis. N Engl Med 1999; 340: 1005-110.

34.- International Liaison Committee on Resuscitation: The International Liaison Committee on Resuscitation (ILCOR) consensus on science with treatment recommendations for pediatric and neonatal patients: neonatal resuscitation. Pediatrics 2006; 117: e978-88. 
35.- Bancalari A, Cleveland C, Kraunik D, et al: Reanimación Neonatal: Experiencia en una Unidad de Neonatología. Rev Chil Ped 2007; 78: 658A.

36.- Bjorklund LJ, Ingimarsson J, Curstedt T, et al: Manual ventilation with a few large breaths al birth compromises the therapeutic effect of subsequent surfactant replacement in immature lambs. Pediatr Res 1997; 42: 348-55.

37.- Morley C, Davis P: Advances in neonatal resuscitation: supporting transition. Arch Dis Child Fetal Neonatal Ed 2008; 93: F334-6.

38.- Davis $P G$, Tan A, O'Donnell CP, et al: Resuscitation of newborn infants with $100 \%$ oxygen or air: a systematic review and meta-analysis. Lancet 2004; 364 : 1329-33.

39.- Bancalari A, Abásolo A, Peñaloza G: ¿Es necesario el oxígeno a flujo libre durante los primeros minutos de vida del RN? Libro Resumen III Congreso Chileno de Neonatología 2008.

40.- Vento M, Asensi M, Sastre J, et al: Resuscitation with room air instead of $100 \%$ oxygen prevents oxidative stress in moderately asphyxiated term neonates. Pediatrics 2001; 107: 642-7.

41.- Soll RF, Morley CJ: Prophylactic versus selective use of surfactant in preventing morbidity and mortality in preterm infants. Cochrane Database Syst Rev 2001; 2: CD000510.

42.- Eichenwald E, Stark AR: Management and Outcomes of Very Low Birth Weight. N Engl J Med 2008; 358 : 1700-11.

43.- Askie LM, Henderson-Smart DJ, Irwig L, Simpson JM: Oxygen-saturation targets and outcomes in extremely preterm infants. N Engl J Med 2003; 349: 959-67.

44.- STOP-ROP Multicenter Study Group: Supplemental therapeutic oxygen for prethreshold retinopathy of prematurity (STOP-ROP), a randomized controlled trial. I: Primary outcomes. Pediatrics 2000; 105: 295 310 .

45.- Avery ME, Tooley WH, Keller JB, et al: Is chronic lung disease in low birth weight infants preventable? A survey of eight centers. Pediatrics 1987; 79: 26-30.

46.- Van Marter LJ, Allred EN, Pagano M, et al: Do clinical markers of barotrauma and oxygen toxicity explain interhospital variation in rates of chronic lung disease? Pediatrics 2000; 105: 1194-201.

47.- Finer $N$ : To intube or not-that is the question: continuos positive airway pressure versus surfactant and extremely low birthweight infants and extremely low birthweight infants. Arch Dis Child Fetal Neonatal Ed 2006; 91: F392-4.

48.- Aly H, Massaro AN, Patel K, El-Mohandes AA: Is it safer to intubate premature infants in the delivery room? Pediatrics 2005; 115: 1660-5.
49.- De Klerk AM, De Klerk RK: Nasal continuous positive airway pressure and outcomes of preterm infants. J Paediatr Child Health 2001; 37: 161-7.

50.- Morley CJ, Davis PG, Doyle Lw, et al: Nasal CPAP or Intubation at Birth for Very Preterm Infants. N Engl J Med 2008; 358: 700-8.

51.- Bancalari A, Bancalari E: Fisiología Respiratoria del Recién Nacido. Pediátrika 1989; 9: 49-54.

52.- Clark R, Gerstmann D, Jobe A, et al: Lung injury in neonates: Causes, strategies for prevention, and longterm consequences. J Pediatr 2001; 139: 478-86.

53.- Mariani G, Cifuentes J, Carlo WA: Randomized trial of permissive hypercapnia in preterm infants. Pediatrics 1999; 104: 1082-8.

54.- Woodgate PG, Davies $M W$ : Permissive hypercapnea for the prevention of morbidity and mortality in mechanically ventilated newborn infants. Cochrane Database Syst Rev 2001; w: CD002061.

55.- Greenough A, Milner AD, Dimitriou G: Synchronised mechanical ventilation for respiratory support in newborn infants. Cochrane Database Syst Rev 2004; 3: CD000456.

56.- Courtney SE, Durand DJ, Asselin JM, et al: High frequency oscillatory ventilation versus conventional mechanical for very low birth weigth infants. N Engl J Med 2002; 347: 643-52.

57.- Johnson AH, Peacock JL, Greenough A, et al: High frecuency oscillatory ventilation for the prevention of chronic lung disease of prematury. N Engl J Med 2002; 347: 633-42.

58.- Bancalari A: Ventilación de alta frecuencia en el recién nacido. Un soporte respiratorio necesario. Rev Chil Pediatr 2003; 74: 475-86.

59.- González A, Sosenko IRS, Chandar J, et al: Influence of infection on patent ductus arteriosus and chronic lung disease in premature infants weighing 1000 grams or less. J Pediatr 1996; 128: 470-8.

60.- Oh W, Poindexter BB, Perritt R, et al: Association between fluid intake and weight loss during the first ten days of life and risk of bronchopulmonary dysplasia in extremely low birth weight infants. J Pediatr 2005; 147: 786-90.

61.- Schmidt B, Roberts RS, Fanaroff A, et al, and the TIPP investigators: Indomethacin prophylaxis, patent ductus arteriosus and the risk of bronchopulmonary dysplasia: further analyses from the trial of indomethacin prophylaxis in preterms (TIPP). J Pediatr 2006; 148: 730-4.

62.- Blakely ML, Kennedy KA, Lally Kp, Tyson JE: Intravenous indomethacin for symptomatic patent ductus arteriosus in preterm infants. The Cochrane Database of Systematic Review 2002; 1: CD003479.

63.- Bancalari E, Claure N, González A: Patent ductus 
arteriosus and respiratory outcome in premature infants. Biol Neonate 2005; 88: 192-201.

64.- Collard KJ, Godeck S, Holley JE, Quinn MW: Pulmonary antioxidant concentrations and oxidative damage in ventilated premature babies. Arch Dis Child Fetal Neonatal Ed 2004; 89: F412-6.

65.- Ahola T, Lapatto R, Raivio KO, et al: N-acetylcysteine does not prevent bronchopulmonary dysplasia in immature infants: a randomized controlled trial. J Pediatr 2003; 143: 713-9.

66.- Davis JM, Parad RB, Michele T, Allred E, Price A, Rosenfeld $W$ : Pulmonary outcome at 1 year corrected age in premature infants treated at birth with recombinant human $\mathrm{CuZn}$ superoxide dismutase. Pediatrics 2003; 111: 469-76.

67.- Schreiber MD, Gin-Mestan K, Marks JD, Huo D, Lee $G$, Srisuparp $P$ : Inhaled nitric oxide in premature infants with the respiratory distress syndrome. N Engl J Med 2003; 349: 2099-107.

68.- Ballard RA, Truog WE, Cnaan A, et al: Inhaled nitric oxide in preterm infants undergoing mechanical ventilation. N Engl J Med 2006; 355: 343-53.

69.- Kinsella JP, Cutter GR, Walsh WR, et al: Early inhaled nitric oxide therapy in premature newborns with respiratory failure. N Engl J Med 2006; 355: 354-64.

70.- Mercier JC, Hummler H, Durmeyer X, et al: The Effects of Inhaled Nitric Oxide on the Development of Bronchopulmonary Dysplasia in Preterm Infants: The EUNO Clinical Trial. 49 Congreso de la Sociedad Europea de Investigación Pediátrica y II Congreso de la Academia de Pediatría Europea. Nice, Francia, Octubre 24-28; 2008.

71.- Schelonka RL, Katz B, Waites KB, Benjamín DK Jr: Critical appraisal of the role of Ureaplasma in the development of bronchopulmonary dysplasia with metaanalytic techniques. Pediatr Infect Dis J 2005; 24: 1033-9.

72.- Jonsson B, Rylander M, Faxelius G: Ureaplasma urealyticum, erythromycin and respiratory morbidity in high-risk preterm neonates. Acta Paediatr 1998; 87: 1079-84.

73.- Lyon AJ, McColm J, Middlemist L, Fergussons S, McIntosh N, Ross PW: Randomised trial of erythromycin on the development of chronic lung disease in preterm infants. Arch Dis Child Fetal Neonatal Ed 1998; 78: F10-4.

74.- Baier RJ, Loggins J, Kruger T: Failure of erythromycin to eliminate airway colonization with Ureaplasma urealyticum in very low birth weight infants. BMC Pediatr 2003; 3: 10.
75.- Tin $W$, Walter $S$, Lacamp C: Oxygen monitoring in preterm babies: too high, too low? Pediatric Respiratory Reviews 2003; 4: 9-14.

76.- Tin W: Optimal Oxygen Saturation for Preterm Babies. Biol Neonate 2004; 85: 319-25.

77.- Greenough A, Alexander J, Burgess $S$, et al: High versus restricted use of home oxygen therapy, health care utilization and the cost of care in chronic lung disease infants. Eur J Pediatr 2004; 163: 292-6.

78.- Brion LP, Primhak RA: Intravenous or enteral loop diuretics for preterm infants with (or developing) chronic lung disease. Cochrane Database Syst Rev 2002; 1: CD001453.

79.- Brion LP, Primhak RA, Ambrosio-Pérez I: Diuretics acting on the distal renal tubule for preterm infants with (or developing) chronic lung disease. Cochrane Database Syst Rev 2002; 1: CD001817.

80.- Brion LP, Primhak RA, Yong $W$ : Aerosolized diuretics for preterm infants with (or developing) chronic lung disease. Cochrane Database Syst Rev 2006; 3: CD001694.

81.- Baraldi E, Filippone M: Chronic Lung Disease after Premature Birth. N Engl J Med 2007; 357: 1946-55.

82.- Goldman SL, Gerhardt T, Sonni R, et al: Early prediction of chronic lung disease by pulmonary function testing. J Pediatr 1983; 120: 613-7.

83.- Allen J, Zwerdling R, Ehrenkranz R: Statement on the care of the child with chronic lung disease of infancy and childhood. Am J Respir Crit Care Med 2003; 168: 356-96.

84.- Davis JM, Sinkin RA, Aranda JV: Drug therapy for bronchopulmonary dysplasia. Pediatr Pulmonol 1990; 8: $117-25$.

85.- Schmidt B: Methylxantine therapy for apnea of prematurity: evaluation of treatment benefits and risks at age 5 years in the international Caffeine por Apnea of Prematurity (CAP) trial. Biol Neonate 2005; 88: 208-13.

86.- Lister P, Iles R, Shaw B: Inhaled steroids for neonatal chronic lung disease. Cochrane Database Syst Rev 2000; 002311.

87.- Wang EE, Tang NK: Inmonuglobulin for preventing respiratory syncytial virus infection. Cochrane Database Syst Rev 2000; 001725.

88.- Meissner HC, Long SS and American Academy of Pediatrics: Committee on Infectious Diseases and Committee on Fetus and, Newborn. Revised indications for the use of palivizumab and respiratory syncytial virus immune globulin intravenous for the prevention of respiratory syncytial virus infections. Pediatrics 2003; 112: 1447-52. 